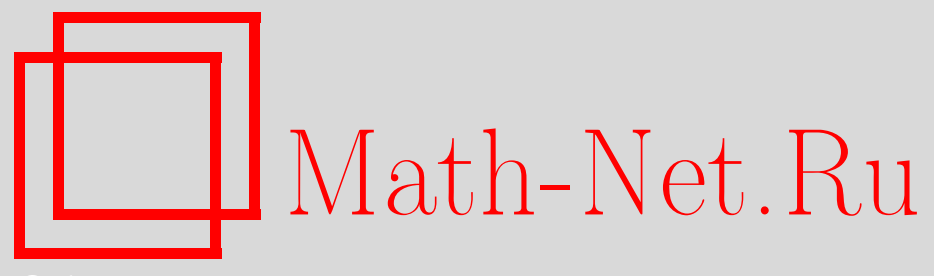

Д. Н. Туляков, Система рекуррентных соотношений для рациональных аппроксимациий постоянной Эйлера, $M a$ тем. заметки, 2009, том 85, выпуск 5, 782-787

DOI: https://doi.org/10.4213/mzm5260

Использование Общероссийского математического портала Math-Net.Ru подразумевает, что вы прочитали и согласны с пользовательским соглашением http://www . mathnet.ru/rus/agreement

Параметры загрузки:

IP : 35.173 .219 .149

26 апреля 2023 г., 16:03:48

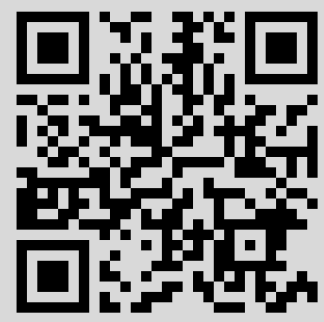


Том 85 выпуск 5 май 2009

УДК 517

\section{Система рекуррентных соотношений для рациональных аппроксимациий постоянной Эйлера}

\section{Д. Н. Туляков}

Получена система рекуррентных соотношений для рациональных аппроксимаций постоянной Эйлера, обобщающая рекуррентные соотношения, полученные ранее Аптекаревым с соавторами. Рекуррентные соотношения этой системы имеют старший коэффициент единица, что позволяет контролировать целочисленность генерируемых чисел.

Библиография: 7 названий.

1. Введение. В сборнике статей [1] получено рекуррентное соотношение для некоторых рациональных аппроксимаций постоянной Эйлера

$$
\gamma:=\lim _{n \rightarrow \infty}\left(\sum_{k=1}^{n} \frac{1}{k}-\ln (n)\right) .
$$

Это соотношение

$$
\begin{gathered}
(16 n-15) q_{n+1}=\left(128 n^{3}+40 n^{2}-82 n-45\right) q_{n}-n^{2}\left(256 n^{3}-240 n^{2}+64 n-7\right) q_{n-1} \\
+n^{2}(n-1)^{2}(16 n+1) q_{n-2}
\end{gathered}
$$

с начальными условиями

$$
\begin{array}{lll}
p_{0}=0, & p_{1}=2, & p_{2}=31, \\
q_{0}=1, & q_{1}=3, & q_{2}=50,
\end{array}
$$

генерирует числители $p_{n}$ и знаменатели $q_{n}$ аппроксимаций, для которых справедливы следующие асимптотические формулы:

$$
\begin{aligned}
q_{n} & =(2 n) ! \frac{e^{\sqrt{2 n}}}{\sqrt[4]{n}}\left(\frac{1}{\sqrt{\pi}(4 e)^{3 / 8}}+O\left(n^{-1 / 2}\right)\right), \\
p_{n}-\gamma q_{n} & =(2 n) ! \frac{e^{-\sqrt{2 n}}}{\sqrt[4]{n}}\left(\frac{2 \sqrt{\pi}}{(4 e)^{3 / 8}}+O\left(n^{-1 / 2}\right)\right) .
\end{aligned}
$$

Формальное доказательство (1.1)-(1.3) см. в [2]-[5].

В настоящей работе мы получим систему рекуррентных соотношений (с единичным старшим коэффициентом) для величин $p_{n}$ и $q_{n}$ из (1.1)-(1.3).

Работа выполнена при поддержке Российского фонда фундаментальных исследований (грант № 08-01-00179), программы "Ведущие научные школы" (грант № НШ-3906.2008.1) и программы № $1 \mathrm{OMH}$ PAH.

(C) Д. Н. Туляков, 2009 
Справедлива

Теорема 1. Пусть последовательность чисел $\widetilde{p}_{n}$ и $\widetilde{q}_{n}$ определена системой рекуррентных соотношений

$$
\begin{aligned}
\widetilde{q}_{4 n-1} & =n \widetilde{q}_{4 n-3}+\widetilde{q}_{4 n-2}, \\
\widetilde{q}_{4 n} & =n \widetilde{q}_{4 n-4}-n \widetilde{q}_{4 n-3}+3 \widetilde{q}_{4 n-2}, \\
\widetilde{q}_{4 n+1} & =n \widetilde{q}_{4 n-1}+(n+1) \widetilde{q}_{4 n}, \\
\widetilde{q}_{4 n+2} & =n \widetilde{q}_{4 n-2}+(2 n+1)^{2} \widetilde{q}_{4 n}-n \widetilde{q}_{4 n+1},
\end{aligned}
$$

с начальными условиями

$$
\begin{array}{lll}
\widetilde{p}_{0}=0, & \widetilde{p}_{1}=1, & \widetilde{p}_{2}=1, \\
\widetilde{q}_{0}=1, & \widetilde{q}_{1}=1, & \widetilde{q}_{2}=1 .
\end{array}
$$

Тогда для $p_{n}$ и $q_{n}$ из (1.1)-(1.3) справедливо

$$
p_{n}=\widetilde{p}_{4 n}, \quad q_{n}=\widetilde{q}_{4 n} .
$$

Отметим, что из (1.4)-(1.5) следует целочисленность числителей $p_{n}$ и знаменателей $q_{n}$ аппроксимаций постоянной Эйлера, причем это важное следствие непосредственно из (1.1)-(1.3) не вытекало.

2. Рекуррентные соотношения для многочленов, задаваемых формулой Родрига. Стартовой точкой в [1] для получения рекуррентных соотношений (1.1)-(1.3) были интегральные представления

$$
p_{n}-\gamma q_{n}=\int_{0}^{\infty} Q_{n}(x) e^{-x} \ln (x) d x, \quad q_{n}=\int_{0}^{\infty} Q_{n}(x) e^{-x} d x,
$$

где многочлены $Q_{n}$ задаются следующей обобщенной формулой Родрига:

$$
Q_{n}(x)=\frac{1}{(n !)^{2}} \frac{e^{x}}{x-1}\left(\frac{d}{d x}\right)^{n} x^{n}\left(\frac{d}{d x}\right)^{n}(x-1)^{2 n+1} x^{n} e^{-x} .
$$

Эти многочлены являются частным случаем приведенных в работе [6] многочленов

$$
Q_{[n, m]}(x)=w_{1}^{-1} \frac{d^{m}}{d x^{m}}\left[w_{1} x^{m} w_{0}^{-1} \frac{d^{n}}{d x^{n}}\left[w_{0} x^{n}(1-x)^{(n+m)}\right]\right],
$$

где весовые функции $w_{j}$ имеют вид

$$
w_{j}(x)=x^{a_{j}}(1-x)^{\alpha} e^{\beta x}, \quad j=0,1 .
$$

Многочлены $Q_{n}$ в $(2.1),(2.2)$ (с точностью до нормировки) получаются из (2.3), полагая

$$
m=n, \quad a_{0}=a_{1}=0, \quad \alpha=-\beta=1 .
$$

В свою очередь, многочлены $Q_{(n, n, m, m)}^{\left(a_{0}, a_{1}, \alpha, \beta\right)}(x):=Q_{[n, m]}(x)$ были приведены в [6] как пример совместно ("multiple") ортогональных многочленов

$$
Q_{\vec{n}}(x)=Q_{(n, n, m, m)}^{\left(a_{0}, a_{1}, \alpha, \beta\right)}(x), \quad \vec{n}:=\left(n_{1}, n_{2}, n_{3}, n_{4}\right) \in \mathbb{N}^{4}, \quad \operatorname{deg} Q_{\vec{n}} \leqslant|\vec{n}|:=\sum_{j=1}^{4} n_{j},
$$


удовлетворяющих следующей системе из четырех соотношений ортогональности с весами $(2.4)$, взятыми на отрезках $\Delta_{1}=[0 ; 1], \Delta_{2}=[1 ; \infty)$ :

$$
\int_{\Delta_{i}} Q_{\vec{n}}(x) x^{\nu} w_{j}(x) d x=0, \quad \nu=0, \ldots, n_{2 j+i}-1, \quad j=0,1, \quad i=1,2 .
$$

Отметим, что при стремлении значений параметров к $(2.5)$ и неравенствах $n_{i+2} \leqslant n_{i}$ для предельных $Q_{\vec{n}}$ имеют место соотношения ортогональности (2.6) с весами

$$
w_{0}(x)=(1-x) e^{-x}, \quad w_{1}(x)=(1-x) \ln (x) e^{-x} .
$$

Известно (см., например, [7]), что $p$ соотношений ортогональности могут порождать для совместно ортогональных многочленов $(p+2)$-членные рекуррентные соотношения. В рассматриваемой ситуации многочленов (2.6) речь идет о рекурсиях, связывающих шесть последовательных многочленов из так называемой "step-line":

$$
\ldots, Q_{\vec{n}-\vec{e}_{4}}, Q_{\vec{n}-\vec{e}_{4}+\vec{e}_{1}}, Q_{\vec{n}-\vec{e}_{4}+\vec{e}_{2}}, Q_{\vec{n}-\vec{e}_{4}+\vec{e}_{3}}, Q_{\vec{n}}, Q_{\vec{n}+\vec{e}_{1}}, \ldots,
$$

где

$$
\vec{e}_{1}=(1,0,0,0), \quad \vec{e}_{2}=(1,1,0,0), \quad \vec{e}_{3}=(1,1,1,0), \quad \vec{e}_{4}=(1,1,1,1) .
$$

Причем, чтобы определять все многочлены вдоль линии (2.7), необходим набор из четырех последовательных рекурсий. Явный вид коэффициентов четырех шестичленных рекуррентных соотношений из такого набора был получен в работе Христофорова [2]. Однако ввиду того, что среди набора многочленов (2.7) встречаются не только многочлены с индексами $\vec{n}=(n, n, m, m)$, определяемые $(2.3)$, эти рекуррентные соотношения содержат коэффициенты с "трансцендентной" зависимостью от входных параметров. В работе Боголюбского [3] при значении параметров (2.5) найдены рекуррентные соотношения с рациональными по $n$ и $z$ коэффициентами, связывающие только "родриговы" многочлены многомерного "step-line" - система из двух семичленных рекуррентных соотношений, а также одно восьмичленное рекуррентное соотношение. Эти рекуррентные соотношения хороши для компьютерных экспериментов и компьютерных доказательств (с помощью них и была открыта и доказана в [4] рекурсия (1.1), (1.2)), однако аналитическое их использование не представляется возможным ввиду громоздкости их коэффициентов.

В настоящей работе для получения системы рекуррентных соотношений (1.4) мы также используем соотношения (2.1) с подстановкой многочленов, определяемых формулами Родрига (2.3). Мы выводим новый набор из четырех шестичленных соотношений, который в отличие от рекуррентных соотношений работы [2] связывает только многочлены вида (2.3) - для смежных параметров $a_{j}$ и $a_{j}+1, j=1,2$, в $(2.3),(2.4)$ и, как результат, коэффициенты этих рекуррентных соотношений превращаются в рациональные функции от входных параметров - $\left(n, m, a_{j}, \alpha, \beta\right)$. Обозначим

$$
\begin{aligned}
Q_{4 k} & =Q_{(k, k, k+t, k+t)}^{a, b, \alpha, \beta}(x), \\
Q_{4 k+1} & =x Q_{(k, k, k+t, k+t)}^{a+1, b+1, \beta}(x), \\
Q_{4 k+2} & =Q_{(k+1, k+1, k+t, k+t)}^{a, b, \alpha}(x), \\
Q_{4 k+3} & =x Q_{(k+1, k+1, k+t, k+t)}^{a+1, b+1, \alpha, \beta}(x) .
\end{aligned}
$$




\section{Справедлива}

ТЕорема 2. Для последователъного определения многочленов $\left\{Q_{n}\right\}_{n=0}^{\infty}$ из (2.8), (2.3) имеют место рекуррентные соотношения

$$
\begin{aligned}
x Q_{(m, m, n, n)}^{a+1, b+1, \alpha, \beta}(x)=( & \left.c_{m n}^{(15)} x+c_{m n}^{(14)}\right) Q_{(m, m, n, n)}^{a, b, \alpha, \beta}(x)+c_{m n}^{(13)} x Q_{(m, m, n-1, n-1)}^{a+1, b+1, \alpha),}(x) \\
& +c_{m n}^{(12)} Q_{(m, m, n-1, n-1)}^{a, b, \alpha, \beta}(x)+c_{m n}^{(11)} x Q_{(m-1, m-1, n-1, n-1)}^{a+1, b+1, \alpha, \beta}(x) \\
& +c_{m n}^{(10)} Q_{(m-1, m-1, n-1, n-1)}^{a, b, \alpha, \beta}(x) \\
Q_{(m+1, m+1, n, n)}^{a, b, \alpha, \beta}(x)=( & \left.c_{m n}^{(25)} x+c_{m n}^{(24)}\right) x Q_{(m, m, n, n)}^{a+1, b+1, \alpha, \beta}(x)+c_{m n}^{(23)} Q_{(m, m, n, n)}^{a, b, \alpha, \beta}(x) \\
& +c_{m n}^{(22)} x Q_{(m, m, n-1, n-1)}^{a+1, b+1, \alpha, \beta}(x)+c_{m n}^{(21)} Q_{(m, m, n-1, n-1)}^{a, b, \alpha, \beta}(x) \\
& +c_{m n}^{(20)} x Q_{(m-1, m-1, n-1, n-1)}^{a+1, b+1, \alpha, \beta}(x) \\
& +C_{m n}^{(2)} Q_{(m-1, m-1, n-1, n-1)}^{a, b, \alpha, \beta}(x) \\
x Q_{(m+1, m+1, n, n)}^{a+1, b+1, \alpha, \beta}(x)=( & \left.c_{m n}^{(35)} x+c_{m n}^{(34)}\right) Q_{(m+1, m+1, n, n)}^{a, b, \alpha, \beta}(x)+c_{m n}^{(33)} x Q_{(m, m, n, n)}^{a+1, b+1, \alpha, \beta}(x) \\
& +c_{m n}^{(32)} Q_{(m, m, n, n)}^{a, b, \alpha, \beta}(x)+c_{m n}^{(31)} x Q_{(m, m, n-1, n-1)}^{a+1, b+1, \alpha, \beta}(x) \\
& +c_{m n}^{(30)} Q_{(m, m, n-1, n-1)}^{a, b, \alpha, \beta}(x), \\
Q_{(m+1, m+1, n+1, n+1)}^{a, b, \alpha, \beta}(x)=( & \left.c_{m n}^{(45)} x+c_{m n}^{(44)}\right) x Q_{(m+1, m+1, n, n)}^{a+1, b+1, \alpha, \beta}(x)+c_{m n}^{(43)} Q_{(m+1, m+1, n, n)}^{a, b, \alpha, \beta}(x) \\
& +c_{m n}^{(42)} x Q_{(m, m, n, n)}^{a+1, b+1, \alpha, \beta}(x)+c_{m n}^{(41)} Q_{(m, m, n, n)}^{a, b, \alpha, \beta}(x) \\
& +c_{m n}^{(40)} x Q_{(m, m, n-1, n-1)}^{a+1, b+1, \alpha, \beta}(x)+C_{m n}^{(4)} Q_{(m, m, n, n-1, n-1)}^{a, b, \alpha, \beta}(x),
\end{aligned}
$$

где коэффициенты первых двух соотношений имеют вид

$$
\begin{aligned}
c_{m n}^{(15)}= & 1, \\
c_{m n}^{(14)} \beta= & m+n, \\
c_{m n}^{(13)} \beta= & -2 m^{2}-3 m n-2 n^{2}-(\alpha+a) m-(\alpha+b) n, \\
c_{m n}^{(12)} \beta^{2}= & \beta\left(m^{2}+m n+n^{2}+a m+b n\right)+m(m+a-b)(2 m+n+a+\alpha), \\
c_{m n}^{(11)} \beta^{2}= & -m(m+a-b)[(m+a) \beta+(2 m+n+a+\alpha)(2 m+n+a+\alpha-1)], \\
c_{m n}^{(10)} \beta^{2}= & m(m+a-b)(m+a)(\beta+2 m+n+a+\alpha), \\
c_{m n}^{(25)}= & \beta, \\
c_{m n}^{(24)}= & 3 m+2 n+a+\alpha+2-\beta, \\
c_{m n}^{(23)} \beta= & -\beta(m+a+1)-2 m^{2}+3 m n+2 n^{2}+(\alpha+a+1) m+(\alpha+b+1) n, \\
c_{m n}^{(22)} \beta= & -\beta(m+n)(n+m+\alpha)+4(m+n)\left(m^{2}+m n+n^{2}\right)+2 m^{2}+3 m n+2 n^{2} \\
& \quad+(\alpha+a) m(4 m+3 n+\alpha+a+1)+(\alpha+b) n(4 n+3 m+\alpha+b+1), \\
c_{m n}^{(21)} \beta^{2}= & -\beta[(m+n+a) m(m+a+1)+(m+n)(n+b)(m+2 n+b+\alpha+1)] \\
& \quad-m(m+a-b)(2 m+n+a+\alpha+1)(2 m+n+a+\alpha), \\
c_{m n}^{(20)} \beta^{2}= & m(m+a-b)(2 m+n+a+\alpha)(2 m+n+a+\alpha)^{2}-1-(n+\alpha-a-1) \beta, \\
C_{m n}^{(2)} \beta^{2}= & -m(m+a-b)(m+a)(m+a+1) \beta \\
& \quad(2 m+n+a+\alpha+1)(2 m+n+a+\alpha),
\end{aligned}
$$


а коэфбициенты двух последующих соотношений определяются из равенств

$$
C_{m, n}^{(4)}(a, b)=C_{n+1, m}^{(2)}(b, a), \quad c_{m, n}^{(j+2, k)}(a, b)=c_{n+1, m}^{(j, k)}(b, a), \quad j=1,2, \quad k=0, \ldots, 5 .
$$

Сделаем несколько замечаний.

1) Второе и четвертое рекуррентные соотношения теоремы 2 содержат семь членов, однако из них легко получить 6-членные соотношения, так как $c_{m n}^{(10)} \neq 0$, $c_{m n}^{(30)} \neq 0$.

2) С учетом возможности придать соотношениям 6-членный вид существует пять линейно независимых решений-последовательностей, одна из которых как раз $\left\{Q_{n}\right\}$ (см. (2.8)). В качестве четырех других можно взять функции второго рода относительно каждой из четырех мер ортогональности.

3) О доказательстве теоремы 2. Из системы четырех шестичленных рекуррентных соотношений работы [2], определяющих многочлены "step-line" - (2.7), можно исключить "неродриговские" многочлены с помощью соотношений

$$
\begin{aligned}
& Q_{(m+1, m, n, n)}^{a, b, \beta}(x)=k_{m n}^{(1)} x Q_{(m, m, n, n)}^{a+1, b+1, \alpha}(x)+k_{m n}^{(2)} Q_{(m, m, n, n)}^{a, b, \alpha, \beta}(x), \\
& Q_{(m, m, n+1, n)}^{a, b, \alpha, \beta}(x)=k_{m n}^{(3)} x Q_{(m, m, n, n)}^{a+1, b+1, \alpha, \beta}(x)+k_{m n}^{(4)} Q_{(m, m, n, n)}^{a, b, \alpha, \beta}(x),
\end{aligned}
$$

где $k_{m n}^{(i)}$ - некоторые константы. При этом, так как $k_{m n}^{(1)} \neq 0, k_{m n}^{(3)} \neq 0$, можно в каждом соотношении выражать многочлен наибольшей степени через остальные, что приведет нас к рекуррентным соотношениям теоремы 2. Выражения для коэффициентов этих рекурсий проще получать непосредственно. Вычисляя семь старших коэффициентов полиномов $Q_{(m, m, n, n)}^{a, b, \alpha, \beta}(x)$ и приравнивая коэффициенты при различных степенях $x$ слева и справа от знаков равенств в рекурсиях теоремы 2 , получаем линейные системы. Решая их, находим явный вид для рекуррентных коэффициентов.

4) О доказательстве теоремы 1. Рассматриваем последовательность многочленов $(2.8)$ при значениях параметров $(2.5)$, т.е. $\left\{Q_{n}(x)\right\}_{n=0}^{\infty}$, где

$$
\begin{aligned}
Q_{4 k}(x) & =Q_{(k, k, k, k)}^{0,0,1,-1}(x), & & Q_{4 k+1}(x)=x Q_{(k, k, k, k)}^{1,1,1,-1}(x), \\
Q_{4 k+2}(x) & =Q_{(k+1, k+1, k, k)}^{0,0,1,-1}(x), & & Q_{4 k+3}(x)=x Q_{(k+1, k+1, k, k)}^{1,1,1,-1}(x) .
\end{aligned}
$$

Определяем с помощью этих многочленов числа $\widetilde{q}_{n}, \widetilde{p}_{n} \in \mathbb{Q}$ :

$$
\begin{aligned}
\widetilde{p}_{n}-\gamma \widetilde{q}_{n} & =\frac{1}{[n / 4] ![(n+2) / 4] !} \int_{0}^{\infty} Q_{n}(x) \ln (x) e^{-x} d x \\
\widetilde{q}_{n} & =\frac{1}{[n / 4] ![(n+2) / 4] !} \int_{0}^{\infty} Q_{n}(x) e^{-x} d x
\end{aligned}
$$

Рекуррентные соотношения теоремы 1 для этих чисел получаются и обосновываются методами работы [4].

В заключение автор выражает благодарность А. И. Аптекареву за полезные советы и обсуждения в процессе работы над этой статьей. 


\section{СПИСОК ЦИТИРОВАННОЙ ЛИТЕРАТУРЫ}

[1] Рациональные приближения постоянной Эйлера и рекуррентные соотношения, Сборник статей, Совр. пробл. матем., 9, ред. А. И. Аптекарев, МИАН, М., 2007, 84 с..

[2] Д. В. Христофоров, "Рекуррентные соотношения для аппроксимаций Эрмита-Паде некоторой системы из четырех функций марковского и стилтьесовского типа", Рациональные приближения постоянной Эйлера и рекуррентные соотношения, Сборник статей, Совр. пробл. матем., 9, МИАН, М., 2007, 11-26.

[3] А. И. Боголюбский, "Рекуррентные соотношения с рациональными коэффициентами для некоторых совместно ортогональных полиномов, задаваемых формулой Родрига", Рациональные приближения постоянной Эйлера и рекуррентные соотношения, Сборник статей, Совр. пробл. матем., 9, МИАН, М., 2007, 27-35.

[4] А.И. Аптекарев, Д.Н. Туляков, "Четырёхчленные рекуррентные соотношения для

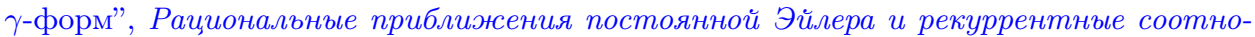
шения, Сборник статей, Совр. пробл. матем., 9, МИАН, М., 2007, 37-43.

[5] А. И. Аптекарев, В. Г. Лысов, "Асимптотика $\gamma$-форм, генерируемых совместно ортогональными многочленами", Рациональные приближения постоянной Эйлера и рекуррентные соотношения, Сборник статей, Совр. пробл. матем., 9, МИАН, М., 2007, $55-62$.

[6] A. I. Aptekarev, A. Branquinho, W. Van Assche, "Multiple orthogonal polynomials for classical weights", Trans. Amer. Math. Soc., 355:10 (2003), 3887-3914.

[7] В. А. Калягин, "Аппроксимации Эрмита-Паде и спектральный анализ несимметричных операторов", Матем. сб., 185:6 (1994), 79-100.

Д. Н. Туляков

Институт прикладной математики им. М. В. Келдыша РАН

E-mail: dnt@mail.nnov.ru
Поступило

09.07.2008

Исправленный вариант

20.10 .2008 